\title{
A SIMPLE SCHEME FOR UNMIXING HYPERSPECTRAL DATA BASED ON THE GEOMETRY OF THE N-DIMENSIONAL SIMPLEX
}

\author{
Paul Honeine $^{(1)}$, Cédric Richard $^{(2)}$ \\ (1) Institut Charles Delaunay (UMR CNRS 6279), LM2S, Université de technologie de Troyes, 10010 Troyes, France \\ (2) Laboratoire H. Fizeau (UMR CNRS 6525), Observatoire de la Côte d'Azur, Université de Nice Sophia-Antipolis, 06108 Nice, France \\ paul.honeinedutt.fr, cedric.richardeunice.fr
}

\begin{abstract}
In this paper, we study the problem of decomposing spectra in hyperspectral data into the sum of pure spectra, or endmembers. We propose to jointly extract the endmembers and estimate the corresponding fractions, or abundances. For this purpose, we show that these abundances can be easily computed using volume of simplices, from the same information used in the classical N-Findr algorithm. This results into a simple scheme for unmixing hyperspectral data, with low computational complexity. Experimental results show the efficiency of the proposed method.
\end{abstract}

\section{INTRODUCTION}

Based on observation satellites, remote-sensing with hyperspectral image analysis has gained wide popularity, with applications to mineral exploration, environmental monitoring, and military surveillance. With a large number of measured wavelength bands, each pixel has a complete spectrum. It is usually assumed that each spectrum can be linearly decomposed into spectra, the so-called endmembers, each provided by some pure material. For a given image, the hyperspectral unmixing problem involves, on the one hand the extraction of these pure spectra and, on the other the estimation of their abundances within each pixel. The number of endmembers is often assumed known; otherwise methods such as the one presented in [1] can be used to estimate it.

The first step is the endmember extraction problem. From convex geometry, this problem is equivalent to finding a dataenclosing simplex, as proposed in [2,3]. One of the most popular (automated) algorithms for endmember extraction is Winter's N-Findr [3]. The main driving force behind this algorithm is a simple iterative and scalable scheme. With a random initial set defining the simplex, it seeks to stretch the simplex in order to maximize its volume by visiting each pixel. The N-Findr is a fast endmember extraction technique with low computational cost, making it the most widely used method despite the restriction on belonging endmembers in the image. The second step involves estimating the abundance of each endmember for all pixels. Many techniques have been proposed to estimate the fraction of pure spectra in a given spectrum. The main difficulties reside in constraints on the estimated coefficients in order to represent the physical abundancies, with the sum-to-one and the non-negativity constraints(see $[4,5]$ and references therein).

In this paper, we give a simple algorithm for extracting the endmembers and their respective adundances, jointly in a single step. The proposed approach takes advantage of the well-known N-Findr scheme for endmember extraction. In the same spirit, we show that we can provide an efficient iterative algorithm for estimating the abundancies. Both the endmember extraction and the abundancies estimation are done in a single step, using the concept of barycentric coordinates which are computed from volumes of simplices. This is done without any additional computational cost as opposed to classical least square estimation techniques [5].

\section{The linear (un-)mixing model}

In a hyperspectral image, the hypothesis of a linear mixed model is often used. The spectrum of a pixel is given as a linear combination of some pure spectra, called endmembers. Let $\boldsymbol{x}_{\ell}$ be the spectrum of the $\ell$-th pixel, then the mathematical model is

$$
\boldsymbol{x}_{\ell}=\sum_{i=1}^{n} \alpha_{i} \boldsymbol{x}_{\omega_{i}}+\epsilon,
$$

where endmembers are defined by spectra $\boldsymbol{x}_{\omega_{i}}$, assumed represented by some pixels $\left\{\omega_{1}, \omega_{2}, \ldots, \omega_{n}\right\}$, and $\epsilon$ corresponds to the unfitness of the model, often treated as a Gaussian noise. In order for the coefficients $\alpha_{1}, \alpha_{2}, \ldots, \alpha_{n}$ to represent the physical abundance fraction associated to each endmember, two constraints must be imposed on theses coefficients:

- The sum-to-one constraint, called hereafter the equality constraint, with

$$
\sum_{i=1}^{n} \alpha_{i}=1
$$

- The non-negativity constraint, with

$$
\alpha_{1}, \alpha_{2}, \ldots, \alpha_{n} \geq 0
$$


Estimating the coefficients based on the model (1) subject to both constraints requires advanced optimization techniques, as studied for instance in [5]. In this paper, we give a direct scheme to solve the equality-constrained optimization problem, incorporating naturally the endmember extraction procedure. Furthermore, we provide a geometric interpretation to the violation of the non-negativity constraint.

\section{THE METHOD}

Unmixing hyperspectral data based on the linear model (1) involves two tasks: extracting the endmembers $\boldsymbol{x}_{\omega_{i}}$ and computing their coefficients $\alpha_{i}$, for $i=1,2, \ldots, n$, and for the spectrum of each pixel. We begin next with the extraction method, as given in the $\mathrm{N}$-findr scheme [3], and then derive in next paragraph the method to estimation the corresponding coefficients.

But before, we give a definition of the volume of a simplex. Let $\boldsymbol{X}=\left\{\boldsymbol{x}_{\omega_{1}}, \boldsymbol{x}_{\omega_{2}}, \ldots, \boldsymbol{x}_{\omega_{n}}\right\}$ be the set of estimated endmembers, with $\omega_{i} \in\{1,2, \ldots\}$. The oriented volume of the simplex defined by the vertices $\boldsymbol{X}$ is given by

$$
\mathcal{V}_{\boldsymbol{X}}=\frac{1}{(n-1) !} \operatorname{det}\left[\begin{array}{cccc}
1 & 1 & \cdots & 1 \\
\boldsymbol{x}_{\omega_{1}} & \boldsymbol{x}_{\omega_{2}} & \cdots & \boldsymbol{x}_{\omega_{n}}
\end{array}\right] \text {, }
$$

where det is the determinant operator. While taking the absolute value of this expression gives the (conventional, positivevalued) volume of a simplex, the virtues of the oriented (signed) volume will be demonstrated in estimating the coefficients.

\section{Endmember extraction}

We take advantage of the fact that the endmembers define the vertices of a simplex englobing all spectra of the image. Thus, this simplex has the largest volume among all simplices constructed from other spectra. Therefore, one seeks the simplex of largest volume, in an iterative manner by visiting each pixel. At initialization, random pixels are selected as endmembers ${ }^{1}$. The following process is iterated for each pixel, where $\boldsymbol{x}_{\ell}$ is its spectrum: One at a time, each endmember is replaced by the pixel under investigation, and the oriented volume of the resulting simplex is computed. Let

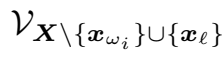

denotes the simplex (oriented) volume of vertices $\boldsymbol{X} \backslash\left\{\boldsymbol{x}_{\omega_{i}}\right\} \cup$ $\left\{\boldsymbol{x}_{\ell}\right\}$, with $\backslash$ the set difference defined by the set $\boldsymbol{X}$ with $\boldsymbol{x}_{\omega_{i}}$ removed and $\boldsymbol{x}_{\ell}$ added. Besides the initial set with simplex volume $\mathcal{V}_{\boldsymbol{X}}$, we have $n$ candidate sets, each defining a sim-

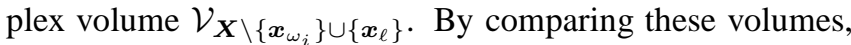
two cases can be distinguished:

\footnotetext{
${ }^{1}$ One may also operate an appropriate selection of the starting set, as proposed for instance in [6]. However, such improvements are beyond the scope of this communication.
}

- if $\max _{i}\left|\mathcal{V}_{\boldsymbol{X} \backslash\left\{\boldsymbol{x}_{\omega_{i}}\right\} \cup\left\{\boldsymbol{x}_{\ell}\right\}}\right|<\left|\mathcal{V}_{\boldsymbol{X}}\right|$, then the initial set of endmembers remains unchanged;

- otherwise, an entry of the initial set is substituted with $\boldsymbol{x}_{\ell}$ to give the new endmembers set. The outgoing spectrum $\omega_{i}$ is identified such as

$$
i=\arg \max \left|\mathcal{V}_{\boldsymbol{X} \backslash\left\{\boldsymbol{x}_{\omega_{i}}\right\} \cup\left\{\boldsymbol{x}_{\ell}\right\}}\right| .
$$

\section{Coefficients estimation}

We are now in a position to compute the coefficients. This is done at the same iteration, using the computed oriented volumes. Given the simplex defined by the vertices $\boldsymbol{x}_{\omega_{1}}, \boldsymbol{x}_{\omega_{2}}, \ldots, \boldsymbol{x}_{\omega_{n}}$, any $\boldsymbol{x}_{\ell}$ can be written as a linear combination of these vertices, i.e.

$$
\boldsymbol{x}_{\ell}=\sum_{i=1}^{n} \alpha_{i} \boldsymbol{x}_{\omega_{i}}
$$

subject to

$$
\sum_{i=1}^{n} \alpha_{i}=1
$$

To solving this constrained optimization problem, we combine the above expressions into the following augmented ${ }^{2}$ linear system

$$
\left[\begin{array}{cccc}
1 & 1 & \cdots & 1 \\
\boldsymbol{x}_{\omega_{1}} & \boldsymbol{x}_{\omega_{2}} & \cdots & \boldsymbol{x}_{\omega_{n}}
\end{array}\right][\boldsymbol{\alpha}]=\left[\begin{array}{c}
1 \\
\boldsymbol{x}_{\ell}
\end{array}\right]
$$

where $\boldsymbol{\alpha}=\left[\begin{array}{llll}\alpha_{1} & \alpha_{2} & \cdots & \alpha_{n}\end{array}\right]^{\top}$ is the vector of coefficients. Using the well-known Cramer's rule, the solution of this linear system can be expressed in terms of the determinants of the above matrix and of matrices obtained from it with one column substituted by the right-hand-side vector. Therefore, we can write

$$
\begin{aligned}
& \alpha_{1}=\frac{\operatorname{det}\left[\begin{array}{cccc}
1 & 1 & \cdots & 1 \\
\boldsymbol{x}_{\ell} & \boldsymbol{x}_{\omega_{2}} & \cdots & \boldsymbol{x}_{\omega_{n}}
\end{array}\right]}{\operatorname{det}\left[\begin{array}{cccc}
1 & 1 & \cdots & 1 \\
\boldsymbol{x}_{\omega_{1}} & \boldsymbol{x}_{\omega_{2}} & \cdots & \boldsymbol{x}_{\omega_{n}}
\end{array}\right]}, \\
& \alpha_{2}=\frac{\operatorname{det}\left[\begin{array}{cccc}
1 & 1 & \cdots & 1 \\
\boldsymbol{x}_{\omega_{1}} & \boldsymbol{x}_{\ell} & \cdots & \boldsymbol{x}_{\omega_{n}}
\end{array}\right]}{\operatorname{det}\left[\begin{array}{cccc}
1 & 1 & \cdots & 1 \\
\boldsymbol{x}_{\omega_{1}} & \boldsymbol{x}_{\omega_{2}} & \cdots & \boldsymbol{x}_{\omega_{n}}
\end{array}\right]} \\
& \alpha_{n}=\frac{\operatorname{det}\left[\begin{array}{cccc}
1 & 1 & \cdots & 1 \\
\boldsymbol{x}_{\omega_{1}} & \boldsymbol{x}_{\omega_{2}} & \cdots & \boldsymbol{x}_{\ell}
\end{array}\right]}{\operatorname{det}\left[\begin{array}{cccc}
1 & 1 & \cdots & 1 \\
\boldsymbol{x}_{\omega_{1}} & \boldsymbol{x}_{\omega_{2}} & \cdots & \boldsymbol{x}_{\omega_{n}}
\end{array}\right]}
\end{aligned}
$$

\footnotetext{
${ }^{2}$ Using an augmented system has been previously introduced in the literature, with the fixed-point-free transform [7]. To our knowledge, this is the first time that barycentric coordinates are applied to provide a simple optimization scheme.
} 

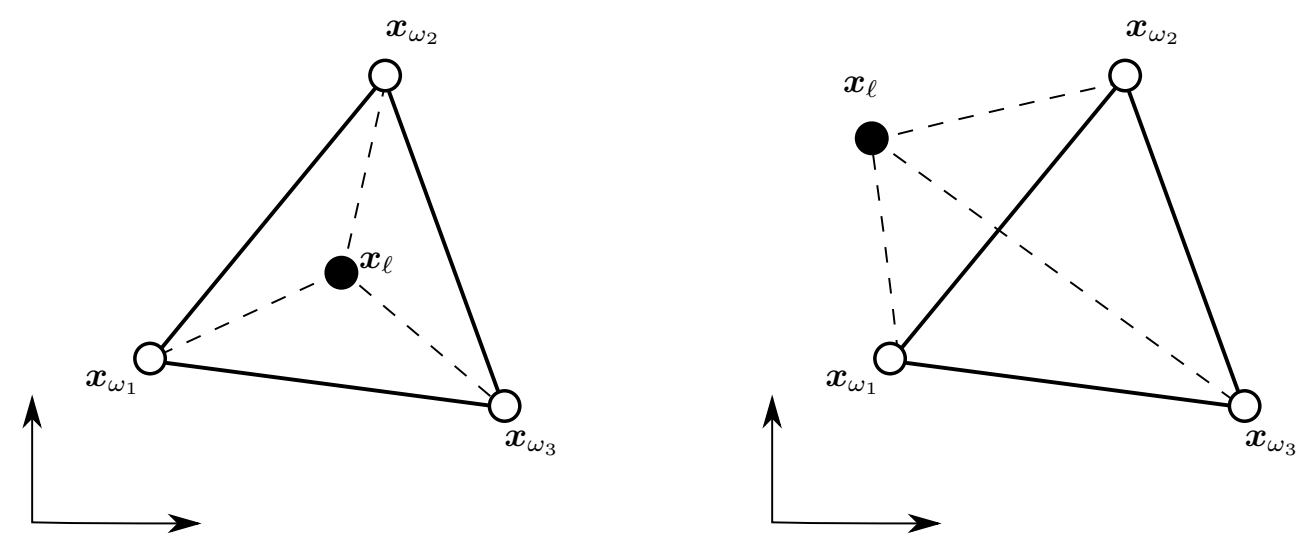

Fig. 1. Illustration of the simplex (here triangle) in a 2-dimensional Euclidean space. Left: the current data $\boldsymbol{x}_{\ell}$ is inside the simplex defined by $\left\{\boldsymbol{x}_{\omega_{1}}, \boldsymbol{x}_{\omega_{1}}, \boldsymbol{x}_{\omega_{1}}\right\}$, and thus all coefficients are non-negative. Right: the data is outside the simplex and thus has at least one negative coefficient.

Thus, each coefficient $\alpha_{i}$ equals a ratio of the oriented volumes of two simplices, the one with vertices $\boldsymbol{X}$ given by endmembers and the one resulting from the latter by substituting vertex $\boldsymbol{x}_{\omega_{i}}$ with $\boldsymbol{x}_{\ell}$. In other words, we have

$$
\alpha_{i}=\frac{\mathcal{V}_{\boldsymbol{X} \backslash\left\{\boldsymbol{x}_{\omega_{i}}\right\} \cup\left\{\boldsymbol{x}_{\ell}\right\}}}{\mathcal{V}_{\boldsymbol{X}}},
$$

for all $i=1,2, \ldots, n$. Since these volumes are initially computed in order to stretch the initial simplex for enclosing most spectra, no additional computational cost is required to evaluate the coefficients at each iteration.

\section{On the non-negativity constraint}

From the literature of geometry of convex sets, the coefficients computed using (5) are called (homogeneous) barycentric coordinates or areal coordinates, defined by the vertices $\boldsymbol{X}$ of the simplex. It is well known that $\boldsymbol{x}_{\ell}$ is inside the simplex if and only if all $\alpha_{i}$ 's are non-negative; otherwise, when it is outside, there exists at least one negative coefficient. The corresponding simplices are illustrated in Fig. 1 for two-dimensional data in two case, whether $\boldsymbol{x}_{\ell}$ lies inside or outside the endmembers simplex.

Therefore, when the solution of the constrained optimization problem (3)-(4) gives at least one negative $\alpha_{i}$, this means that the $\boldsymbol{x}_{\ell}$ is outside the simplex, and thus cannot be written in terms of linear combination of its vertices with both equality and non-negativity constraints. It is worth noting that such limitation is valid for any simplex-based approach. Therefore, this provides a measure of adequacy of the geometric approach, i.e. the simplex englobing the data with the simplex vertices available within the data.

\section{EXPERIMENTATIONS}

In order to illustrate the proposed method, we simulated a synthetic hyperspectral image from a linear combination of three pure materials. These materials are available from the USGS library [8], and correspond to golden grass, red brick, and cedar, with spectra illustrated in Fig. 2 (left). A 128-by128 hyperspectral image is generated from pixels given by the model (1), where $\epsilon$ corresponds to a white Gaussian noise of variance 0.01 . Using the same noisy model with the endmembers, they are incorporated in the image with the canonical coefficients $\{(1,0,0) ;(0,1,0) ;(0,0,1)\}$, as illustrated in Fig. 2 (right).

Most simplex-based methods require a preprocessing dimensionality reduction technique. For this purpose, we apply a classical PCA as suggested in [3]; alternatively, more dedicated methods such as minimum noise fraction technique can be considered. We apply the proposed method with the number of endmembers set to 3 . The resulting largest-volume simplex encloses $71 \%$ of the spectra, following from incorporating noise, not only in the coefficients by also in the endmembers. To measure the performance of the algorithm, we compute the spectral angle error [9] for each pixel, between its initial spectrum and the one computed from the obtained coefficients, of the form

$$
\alpha\left(\boldsymbol{x}_{i}, \boldsymbol{x}_{j}\right)=\cos ^{-1}\left(\frac{\left\langle\boldsymbol{x}_{i}, \boldsymbol{x}_{j}\right\rangle}{\left\|\boldsymbol{x}_{i}\right\|\left\|\boldsymbol{x}_{j}\right\|}\right) .
$$

The histogram of these errors given in Fig. 3 shows a small angular error for the hyperspectral image.

\section{CONCLUSION}

In this paper, we studied the problem of unmixing hyperspectral data. Based on the well-known Winter's N-Findr scheme, we showed that we can jointly operate extraction of endmembers and computing the abundances, using volumes of simplices. 

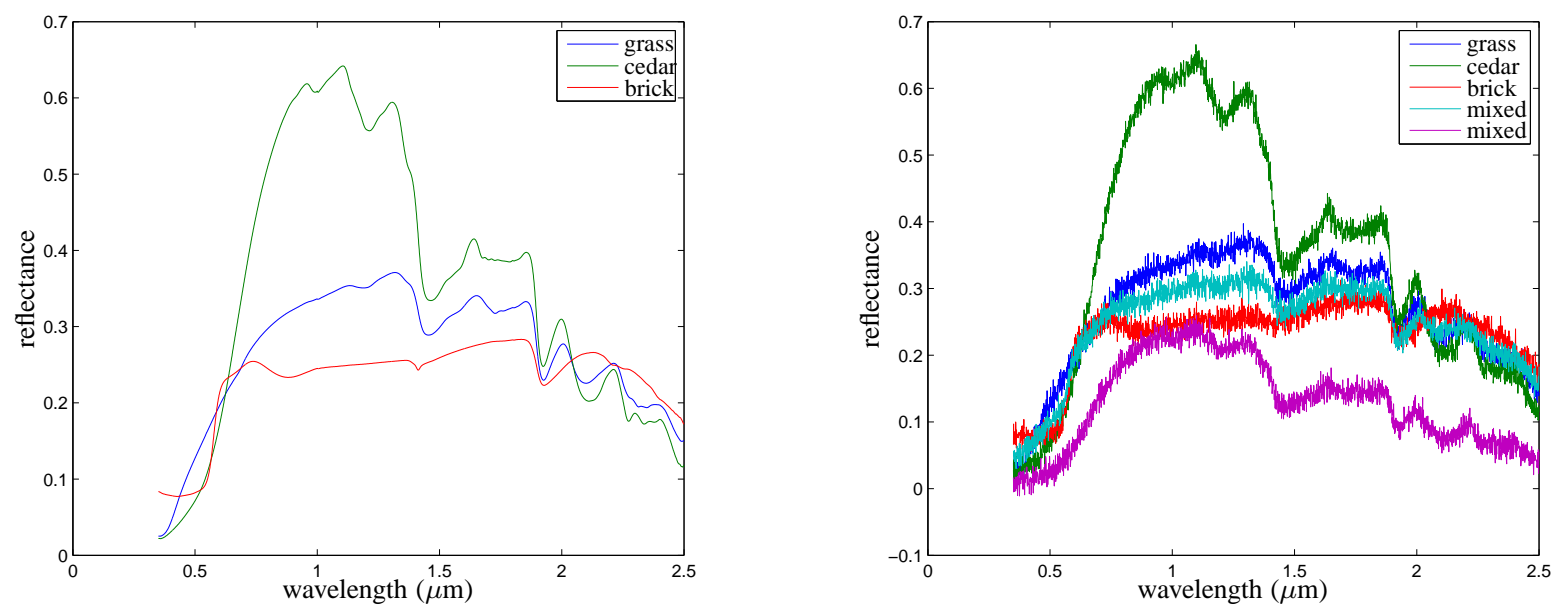

Fig. 2. Endmember spectra: (left) initial spectra and (right) their noisy versions used in generating the hyperspectral image, including two random mixed spectra.

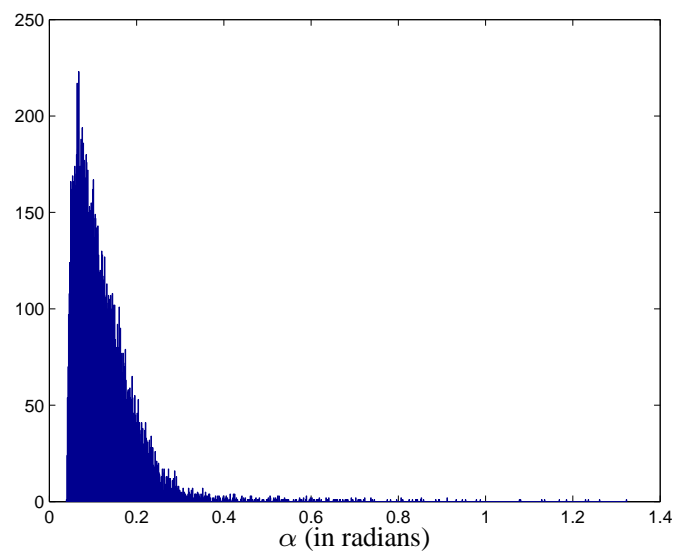

Fig. 3. Histogram of the error using the spectral angle between initial (noise-free) and obtained (noisy) specta.

\section{REFERENCES}

[1] C. Chang and Q. Du, "Estimation of number of spectrally distinct signal sources in hyperspectral imagery," IEEE Trans. Geoscience and Remote Sensing, vol. 42, no. 3, pp. 608-619, March 2004.

[2] J. W. Boardman, "Automating spectral unmixing of aviris data using convex geometry concepts," in Proc. Summ. 4th Annu. JPL Airborne Geosci. Workshop, R. O. Green, Ed., 1994, pp. 11-14.

[3] M. Winter, "N-findr: an algorithm for fast autonomous spectral end-member determination in hyperspectral data," Proceedings of SPIE: Imaging Spectrometry V, vol. 3753, no. 10, 1999.
[4] N. Keshava, J. P. Kerekes, D. Manolakis, and G. A. Shaw, "An algorithm taxonomy for hyperspectral unmixing," in Proceedings of the SPIE, SPIE AeroSense, Algorithms for Multispectral, Hyperspectral, and Ultraspectral Imagery VI, Detection and Identification I, vol. 4049. Orlando, Florida, United States: SPIE, April 2000, pp. 42-63.

[5] D. Heinz and C. Chang, "Fully constrained least squares linear spectral mixture analysis method for material quantification in hyperspectral imagery," IEEE trans on Geoscience and Remote Sensing, vol. 39, no. 3, pp. 529-545, March 2001.

[6] A. Plaza and C.-I. Chang, "An improved n-findr algorithm in implementation," in Proc. SPIE Algorithms and Technologies for Multispectral, Hyperspectral, and Ultraspectral Imagery XI, P. E. Shen, Sylvia S.; Lewis, Ed., vol. 5806, 2005, pp. 298-306.

[7] M. Craig, "Minimum-volume transforms for remotely sensed data," IEEE trans. Geoscience and Remote Sensing, vol. 32, no. 3, pp. 542-552, May 1994.

[8] R. N. Clark and Geological Survey (U.S.), USGS digital spectral library splib06a [electronic resource], rev. sept. 20, 2007. ed. U.S. Geological Survey, Denver, CO, US, 2007.

[9] F. A. Kruse, A. B. Lefkoff, J. W. Boardman, K. B. Heidebrecht, A. T. Shapiro, J. P. Barloon, and A. F. H. Goetz, "The spectral image processing system (SIPS): Interactive visualization and analysis of imaging spectrometer data," Remote Sensing Environ, vol. 2-3, no. 44, pp. 145163, 1993. 